\title{
Collezione di sguardi
}

Introduzione

\section{Matteo Martelli e Marina Spunta}

\section{CpenEdition}

Journals

Edizione digitale

URL: https://journals.openedition.org/cher/653

DOI: $10.4000 /$ cher.653

ISSN: 2803-5992

\section{Editore}

Presses universitaires de Strasbourg

\section{Edizione cartacea}

Data di pubblicazione: 9 juillet 2020

Paginazione: 7-18

ISBN: 979-10-344-0068-3

ISSN: 1968-035X

Notizia bibliografica digitale

Matteo Martelli e Marina Spunta, «Collezione di squardi», reCHERches [Online], 24 | 2020, online dal 20 septembre 2021, consultato il 20 novembre 2021. URL: http://journals.openedition.org/cher/653 ; DOl: https://doi.org/10.4000/cher.653 


\title{
Collezione di sguardi Introduzione
}

\author{
Matteo Martell \\ MARINA SPUNTA ${ }^{1}$
}

Ineluttabile modalità del visibile: se non altro questo, pensiero attraverso gli occhi. J. Joyce, Ulisse (trad. di G. Celati)

Tel 2004, invitato a riflettere sullesperienza e sul significato del progetto $\mathrm{N}$ Viaggio in Italia (Ghirri et al. 1984), Gianni Celati propone un lungo saggio, «Viaggio in Italia con 20 fotografi, 20 anni dopo», entro il quale ripercorre quell'incontro sorprendente, l'itinerario di riflessione compiuto insieme agli artisti e la maniera attraverso la quale quell'avventura diede luogo a «un inedito e saltellante abbecedario del paesaggio italiano» (Valtorta 2013: 22). Lautore ricorda che la sua partecipazione, unico scrittore reclutato nel gruppo, arrivò con una telefonata di Luigi Ghirri, nel 1981, primo momento di un dialogo "così denso da cambiare molte mie idee» (Celati 2008: 134). La novità di quel progetto, e le sue riverberazioni nell'opera di Celati, non si saprebbero in effetti dire in poche parole. Ma è forse proprio questo ciò di cui parla lo scrittore in quelle pagine, quando, rileggendo a distanza di vent'anni un momento per più motivi germinale, mette in dialogo il lavoro di quei fotografi con la propria riflessione sullimmagine, le cui tracce pubbliche risalgono ad almeno due decenni prima di quel famoso 1984.

$\grave{E}$ questo in effetti il senso che possiamo dare al percorso che Celati propone nel saggio sopracitato. Poiché, se da un lato i punti del discorso celatiano si muovono sulla novità di quel viaggio, sulle scelte fatte dal gruppo e sulla maniera di pensare la fotografia come spazio conoscitivo, come ricomposizione

1 Matteo Martelli, Université de Strasbourg, CHER Marina Spunta, University of Leicester. 
dell'immagine «di un luogo, e antropologico e geografico insieme» (Ghirri et al. 1984), d'altro lato, per richiamare questi soggetti Celati pone interrogazioni e riferimenti sui quali lui stesso sta da tempo riflettendo: quando pensa ad alcune ricerche artistiche come quella di Giacometti; ad alcune riflessioni teoriche come quelle di Benjamin e Deleuze; ad alcune esperienze cinematografiche, come quelle di Rossellini, Visconti, Fellini; solo per citare alcuni momenti che entrano in dialogo con quel libro e i suoi autori. Pensando ai fotografi, Celati sta allora anche pensando al proprio rapporto con la fotografia, e più ampiamente con le arti visive e l'universo del visibile. Ed è qui, tra i riferimenti e le interrogazioni che Celati sviluppa, che ci si imbatte in una singolare espressione, forse ripresa da Ghirri, che lo scrittore adopera più o meno a metà del suo racconto. Un'espressione che, nella sua evidenza misteriosa, nel suo esporsi in risonanza e vibrazione, non smetterà di ritrovarsi tra le pagine del presente volume; e che di questo volume, in fondo, è il luogo di nascita.

Quanto afferma Celati è che in quelle fotografie, in alcuni di quegli scatti, ciò che si esprime è «un vedere del vedere» (Celati 2008: 132). Lo scrittore, è chiaro, non sta qui pensando a una vista aumentata, a una maniera di veder meglio, di più, che le immagini di Viaggio in Italia offrirebbero allo spettatore. Non sta nemmeno riflettendo su una possibile traduzione in altro contesto della nota formula d'Italo Calvino, il mondo che guarda il mondo, una sorta di vedere del vedere del mondo. Quello a cui sta pensando, differentemente, è qualcosa che oltrepassa quel libro fotografico, e forse anche quell'esperienza, per toccare alcuni punti essenziali, e a lui cari, della nostra maniera d'osservare e di porci di fronte alle immagini.

Un «vedere del vedere», dunque. Il contesto in cui si inserisce quest'espressione sono alcune scelte che quel gruppo di fotografi ha fatto, in questo caso privilegiando «un impianto ottico» attraverso il quale si «mostra la fotografia come impronta» favorendo «la riflessione sulle immagini» (Celati 2008: 132). Per introdurre il suo discorso, Celati si riferisce a un noto scatto di Mario Cresci, a Salandra, in provincia di Matera (Ghirri et al. 1984: 64). L'immagine è quella di un campo da calcio in terra battuta, alla periferia del paese, su cui qua e là piccoli cespugli erbosi fanno ancora la loro comparsa. Apparentemente un campo poco usato, quasi lasciato a se stesso, non fosse che la linea bianca di gesso della metà campo, con il suo grande cerchio attorno, deve essere stata rifatta da poco, e a mano, vista l'imprecisione con cui il gesso stesso si è depositato sul terreno (su cui qualcuno, forse, deve avere già corso), rendendo quelle linee già come sfocate, sfilacciate, pronte nuovamente a svanire nel tempo.

Noi, come spettatori, siamo su quella linea di metà campo, verticale e al centro dell'immagine. Guardiamo questo strano rettangolo di terra ai margini di non si sa cosa (delle colline sullo sfondo, un terrapieno in primo piano), ma chiuso nel suo recinto, delimitato rispetto a quell'esterno imprecisabile da una rete metallica. Poiché ciò che vediamo (avvertiamo, nella loro trasparenza) sono in effetti due file orizzontali, parallele, di un reticolato probabilmente datato e i pali di sostegno disposti lungo i lati del campo che ritagliano l'immagine, 
appena ma distintamente, nel suo continuare oltre la rete. Quanto ci sta davanti, ricorda Celati, è in effetti una seconda inquadratura all'interno dell'inquadratura fotografica, che in quanto tale «crea un gioco di piani geometrici».

Ora, se lo scrittore fa riferimento a un accorgimento fotografico usato da Cresci come da altri fotografi del gruppo, è perché quella doppia inquadratura interviene nello smarrimento che prova l'osservatore di fronte a una tale fotografia. Interviene portando un dubbio, un'incertezza: che lo sguardo si muova dall'alto in basso, o viceversa, si troverà almeno un istante rallentato da quella rete, dall'inquadratura interna, e dal di qua di quella rete metallica osserverà nuovamente il campo da calcio, forse per pochi attimi, il tempo di sentire quello sguardo, di vedersi guardare dal di qua della rete. Ma guardare cosa?

È questo il punto su cui si sofferma Celati, il fatto che in alcune fotografie di Viaggio in Italia diviene visibile, affiora, qualcosa che accompagna ogni nostro sguardo, se solo sospendiamo per un momento una certa abitudine che ci porta a riconoscere e non a vedere. «Nella loro estrema normalità, queste foto propongono un enigma», scrive Celati, «accennano all'esperienza del vedere come un'esperienza che non può mai essere data per scontata. Il vedere è sempre una ricerca per "vedere", è un'inquietudine per capire cosa vediamo nella mente» (Celati 2008: 128).

Per comprendere quanto Celati sta mettendo a fuoco, possiamo riferirci ad alcune riflessioni di Georges Didi-Huberman, che a questo tema ha dedicato molte pagine. Il mondo che ci sta davanti, come l'opera d'arte, scrive lo studioso francese, non si dà come un'evidenza visibile. Ogni sguardo, per quanto possa essere toccato da ciò che vede, non è semplice ricomposizione di una realtà data (come se di fronte al campo da calcio fotografato da Cresci il nostro vedere si riducesse a un riconoscimento, tautologico, del tipo: è un terreno da gioco di periferia dai colori lionati). L'atto del vedere, differentemente, è un luogo di tensioni che si gioca tra oggetto osservato, colui che guarda, e le maniere stesse del suo sguardo:

[Ni] l'objet, ni le sujet, ni l'acte de voir, jamais ne s'arrêtent à ce qui est visible au sens de ce qui donnerait un terme discernable et adéquatement dénommable [...]. Lacte de voir n'est pas l'acte d'une machine à percevoir le réel en tant que composé d'évidences tautologiques. [...] Voir, c'est toujours une opération du sujet, donc une opération refendue, inquiétée, agitée, ouverte. (Didi-Huberman 1992: 51)

Guardare è dunque sempre un guardare con, attraverso o secondo quanto stiamo guardando, ossia è anzitutto esposizione di qualcosa verso cui ci si espone: un'apertura che presuppone un accoglimento, un attraversamento e un avvolgimento, un'andata e un ritorno, che mette in gioco il soggetto tanto quanto egli stesso si mette in gioco in quell'esposizione; il percepire (il sentire) come provare, un sentirsi-sentire (Nancy 2002: 23).

C’è un secondo esempio, nel testo di Celati, di questo «vedere del vedere». Si tratta di una particolare fotografia di Luigi Ghirri, scattata a Ferrara nel 1981 
(Ghirri et al. 1984: 94). Poche altre fotografie, probabilmente, potrebbero avere un aspetto insieme così materiale e invisibile. Apparentemente è soltanto un muro di mattoni rossi, che copre l'intera inquadratura, lasciando solamente in basso lo spazio d'un marciapiede. Un'immagine piena, verticale, dunque. Una traccia nera, appena pochi centimetri, percorre (scava) quel muro; una traccia rettangolare, forse un vecchio ingresso ora chiuso o una futura apertura, che ha però lasciato l'impronta di una seconda inquadratura. Si direbbe che l'impressione sia quella di trovarsi davanti a qualcosa di frontale piuttosto che a una frontiera, ma ancora una volta invece quell'immagine inquieta, apre, si fa soglia di un "vedere che cerca la sintesi d'un "vedere" mentale [...] in risposta ai nostri desideri» (Celati 2008: 133). La semplice traccia che percorre quel muro è interrogazione, crepa e fenditura dell'immagine stessa, e con essa fessura e inquietudine dello sguardo.

Di fronte a questa fotografia, scrive Celati, capiamo che "ogni immagine è un sistema complesso, che combina le nostre percezioni, i desideri del vedere, i riferimenti alla realtà e i loro riflessi immaginativi, su una superficie che funziona come una casella vuota da riempire con le nostre proiezioni o interpretazioni» (Celati 2008: 133). Celati scrive bene «ogni immagine», non solo quella di Ghirri. Ogni singola immagine che ci si presenta di fronte (e proprio per quel suo stare di fronte) è come una "casella vuota da riempire con le nostre proiezioni». Ecco il «vedere del vedere». Quest'incontro ogni volta inaspettato, inatteso, sorprendente con qualcosa dell'immagine che è già preso, imbrigliato e avvolto dal nostro sguardo (dallo sguardo che è il nostro corpo), qualcosa che altrove Celati chiama con una sua parola guida, affezione. Ciò che ne emerge, aggiunge lo scrittore, è «un punto indicibile» dove «il mondo reale e quello immaginario si riflettono l'uno nell'altro» (Celati 2008: 132).

Questo incontro e congiunzione di due mondi, il loro riflettersi, non significa però specchiarsi, ma entrare in un ritmo dello sguardo, come un ascolto dello sguardo che è vibrazione, rimbalzo, riverbero, altalena. Uno sguardo che come ascolto è allora in primo luogo risonanza: «celle d'un corps sonore pour luimême et celle de la sonorité dans un corps écoutant qui, lui-même, sonne en écoutant» (Nancy 2002: 77). In questa oscillazione, nella compenetrazione di queste oscillazioni sonore e d'ascolto, si gioca qualcosa d'essenziale nel pensiero figurale di Gianni Celati, nella sua maniera di pensare il visibile, l'esterno visibile che si offre a volte come veduta, paesaggio, a volte come pittura, fotografia o cinema. Ogni volta delle linee d'un esterno che ci toccano, in quel legame complesso tra un soggetto che percepisce e ciò che lo riguarda in quello che guarda, proprio perché di fronte a lui, parte di un esterno con cui è in contatto.

Possiamo pensare al "vedere del vedere» come al fil rouge della ricerca sulla visualità e le arti visive di Celati. Lo possiamo seguire nei suoi romanzi, saggi e documentari, o osservando i suoi «incantamenti» di fronte alle sculture pop di Claes Oldenburg, la pittura di Giorgio Morandi, le sculture di Alberto Giacometti, nelle indisciplinate visioni delle slapstick comedies, o ancora nelle collaborazioni e amicizie, con Carlo Gajani, Antonio Faeti, Luigi Ghirri e John 
Berger. Ciò che Celati segue, in ognuna delle sue avventure con le arti visive, sono modi e mondi di racconto, incontri per una collezione di sguardi, ogni volta inediti e sorprendenti.

Ma in ogni sguardo, nella collezione degli sguardi, quel «vedere del vedere» è in primo luogo il tentativo di avvicinarsi e girare «intorno a un punto indicibile» (Celati 2008: 133), a quanto lo scrittore chiama altrove un impensato: qualcosa che affiora e si sottrae al discorso, qualcosa che ci dona meno una chiarezza che una postura, un orientamento. Gilles Deleuze, in uno dei libri cari a Celati, in maniera simile nominava impensabile delle intensità, dei ritmi, dei limiti, un incontro singolare, e fondamentale, nel campo del sensibile, in cui il pensiero trova qualcosa che non può pensare e che non può non essere pensato (Deleuze 1968: 249). Era l'incontro con l'arte, con le affezioni, con ciò che invece di chiederci un riconoscimento ci inquieta, ci pone di fronte a quel punto indicibile delle immagini, come apparizione e guida, misura per orientarci, là dove il discorso non può darci spiegazioni, ma visioni.

La scrittura dello sguardo. Gianni Celati e le arti visive raccoglie una selezione di contributi presentati all'omonimo convegno internazionale tenutosi all'Università di Strasburgo l'8 e il 9 novembre 2018. Nato su iniziativa di Matteo Martelli, il convegno è stato organizzato assieme a Nunzia Palmieri e Marina Spunta, con il sostegno del centro di ricerca Culture et histoire dans l'espace roman (CHER), dell'Istituto Italiano di Cultura di Strasburgo, del Dipartimento di Lettere, Filosofia, Comunicazione dell'Università di Bergamo, della School of Arts dell'Università di Leicester e della Society for Italian Studies. Le due giornate di studi hanno riunito studiosi operanti in diverse discipline e in vari paesi (Italia, Francia, Regno Unito, Stati Uniti), e hanno dato vita a un proficuo dialogo, come ricorda anche Palmieri nella premessa a questo volume, grazie a interventi che hanno offerto nuove prospettive e riletture dell'opera celatiana, come pure dei molti scritti inediti, recentemente catalogati e resi disponibili presso il Fondo Celati, della Biblioteca Panizzi a Reggio Emilia.

L'intento principale del volume, e prima ancora del convegno, è di colmare una lacuna negli studi celatiani: mostrare la centralità della riflessione sulle arti visive - arti figurative, fotografia e cinema - all'interno della sua opera. Se negli ultimi anni vari saggi hanno esplorato soprattutto il Celati cineasta e il rapporto con Ghirri e altri fotografi, solo recentemente l'attenzione di alcuni studiosi si è rivolta verso una lettura più distesa dell'apporto delle arti visive nel pensiero e nel lavoro dell'autore (Palmieri 2017, Martelli 2019). Nessun volume, finora, era però stato interamente dedicato a un ripensamento olistico della scrittura celatiana sullo sguardo, la visione e il «vedere del vedere» nelle sue varie accezioni. Ci siamo proposti di far ciò in un'ottica interdisciplinare e interartistica, che privilegiasse il dialogo tra arti e discipline, e la riflessione teorica su di esse, e che mettesse in luce l'interdipendenza del pensiero celatiano 
su questi temi, a partire dal suo postulare sia l'ascolto che la visione come risonanze, e come forme di sensorialità inscindibili e necessarie al narrare.

La scrittura dello sguardo mostra come nel lavoro di Gianni Celati le arti visive costituiscano al tempo stesso una materia di riflessione e di formazione del pensiero, un oggetto di ricerca critica e artistica, un incontro e uno scambio per pratiche interdisciplinari e scritture ibride. Come emerge dai saggi inclusi nel volume, la riflessione su cinema, fotografia, arti figurative è elemento portante della sua ricerca su un "pensiero figurale» e prende forma in alcune delle tematiche care allo scrittore: le forme della percezione, il concetto d'esperienza e la relazione all'esterno, le interrogazioni sulla condizione del soggetto, la sua presenza e i suoi movimenti nello spazio contemporaneo, la riflessione sull'idea di visione. Sviluppando un'indagine trasversale, questo volume compone i frammenti di un percorso artistico e teorico sui bordi della scrittura di Celati, per studiare la specificità dei contributi e della riflessione sulle arti visive e la loro elaborazione nella sua opera letteraria. Data la pervasività del pensiero celatiano sul visivo e sul visibile, abbiamo concepito un percorso testuale fluido, un insieme di saggi che si rimandano l'un l'altro e che si sviluppano attorno a tre nuclei interdipendenti di pensiero e pratica artistica: le arti visive e la visione, la fotografia e il cinema.

L'interesse di Gianni Celati per ognuno di questi campi artistici si iscrive all'interno di uno sguardo più ampio verso le arti e l'immagine e all'interno di un discorso di continuità fra i codici. In quest'ottica il saggio di Maria Teresa De Palma che abbiamo posto in apertura, dal titolo «Dalla pop art alla pittura antica: visioni, limiti e discontinuo negli scritti sull'arte di Celati», offre un'ampia panoramica e un'attenta rilettura degli scritti celatiani sull'arte, sottolineando la continuità del suo pensiero sulla visione e l'importanza generativa dell'immagine e della pittura per la scrittura celatiana. Per De Palma l'immagine «si configura sempre in Celati come possibilità di evasione da una teoria "fissista" dell'esistere, come luogo di rifiuto di un'estetizzazione forzata e precostituita del mondo, e come portale di una modalità d'essere e di esperire il mondo stesso che sia radicalmente pre-teorica, e invece estesiologica ed emotivamente connotata». In linea con questo approccio, e con un "vedere del vedere» che non tenti di afferrare rigidamente le cose, il contributo di Matteo Martelli riflette sul pensiero celatiano sulla traccia, «lo spazio d'assenza e il negativo del visibile», affrontando temi finora trascurati dalla critica celatiana. Tramite una lettura ravvicinata della sezione finale del saggio "Collezione di spazi», Martelli mette in dialogo il pensiero di Celati con l'arte di Alberto Giacometti e di Rachel Whiteread da un lato, e con gli scritti di Maurice Merleau-Ponty e di Jean-Luc Nancy dall'altro, suggerendo come la riflessione dello scrittore sulla percezione e sulla visione si muova entro una dialettica tra visibile e invisibile attraverso la quale, come nell'opera dell'artista britannica, il vedere si confronta sempre con una latenza, «una possibilità d'essere, una forma di vita intravista, già sempre dispersa nello spazio, compressa nell'interno del suo spazio». 
Con i saggi di Marco Antonio Bazzocchi e di Filippo Milani l'attenzione si sposta sulla luce, sulla pittura e sui paesaggi padani, per evidenziare una consonanza di vedute da un lato con Giorgio Morandi, dall'altro con i dipinti di Turner, a partire dalla luce evanescente (potremmo dire «scoppiata» per usare un aggettivo celatiano) delle sue ultime opere. In linea con la posizione di altri autori qui pubblicati, in "Gianni Celati e Giorgio Morandi: linea, luce, lontananza» Bazzocchi rilegge Verso la foce e Condizioni di luce sulla via Emilia, al fine di sottolineare echi ghirriani, oltre che morandiani, mettendo in luce da un lato la «dissoluzione della prospettiva e [dello] spazio razionalizzante», dall'altro un esterno in cui rimangono solo le apparenze. Questo termine, caro a Celati, secondo Bazzocchi indica «non semplicemente ciò che appare ma ciò che appare a un occhio che ha rinunciato a conoscere ma vuole solo trovare un posto nell'insieme delle cose del mondo» e che tenta di mettere insieme le apparenze per non perdersi nel vuoto, costruendo così il proprio «racconto dell'esterno». Soffermandosi ancora su questi temi, in "La luce "turneriana" dei paesaggi padani» Filippo Milani mette in relazione lo spazio architettonico, anche se latente, dei paesaggi della valle del Po raccontati da Celati con la luce dei dipinti dell'ultimo Turner, letti tramite il «naturalismo informale» di Francesco Arcangeli, e suggerisce che la prosa celatiana intende risensibilizzare lo sguardo alla percezione fenomenologica del mondo. Milani iscrive la scrittura celatiana degli anni Ottanta all'interno della lunga riflessione teorica sulla natura e sul paesaggio e in una tradizione padana, e pone una corrispondenza tra la posizione dello scrittore sullo spazio e sulla visione e la lettura di Arcangeli del romanticismo inglese e di Turner come inafferrabile e ambiguo.

Un altro dialogo finora inesplorato dalla critica celatiana è quello sull'immaginario (pedagogico) e sulla collaborazione tra Celati e Antonio Faeti, primo titolare del rinomato corso di Storia della letteratura per l'infanzia a Bologna dai primi anni Ottanta, oltre che pittore e illustatore. Sfruttando materiale inedito del Fondo Celati, in «Nelle cantine dell'immaginario: su Gianni Celati e Antonio Faeti (1968-1972)» Giacomo Micheletti offre un'attenta lettura di uno dei periodi più fecondi del dialogo (e della lunga amicizia) fra i due artisti, e di documenti poco studiati come il catalogo per Kindertraumdeutung, prima mostra personale di Faeti, presso la Galleria 42 in via D’Azeglio a Bologna, per cui Celati scrive il saggio «Il Qui e l'Altrove», progetto coevo al non terminato Ali Babà. Trapela da quest'analisi una comunanza di passioni per il nonsense e il patwchwork, dall'Alice di Lewis Carroll a Céline a Pinocchio, nel comune intento di «rileggere contropelo la storia dell'immaginario italiano» e non solo, secondo un approccio «archeologico».

In linea con artisti e scrittori a lui affini, come suggerisce il saggio di Spunta, Celati iscrive la sua riflessione sulla fotografia in un più ampio discorso sul «pensare per immagini» che permetta di vedere e descrivere l'esterno con una lingua «necessaria» che si apra all'indeterminatezza. Da uno spoglio degli scritti celatiani, editi e inediti, emerge chiaramente come l'autore veda Luigi Ghirri come sinonimo di fotografia e, più in generale, di un'arte che pone l'«esperienza» 
come modo di «sentire-pensare-immaginare» (Celati s.d.). Quindi non stupisce che molta della critica celatiana si sia concentrata e continui a esplorare la collaborazione e consonanza con Ghirri, che in quegli anni è il perno di numerosi progetti che portano la fotografia italiana al centro di vari dibattiti teorici e artistici (cfr. Spunta e Benci 2017). I saggi inclusi in questo volume partono da questo importante punto di riferimento, continuando a sviluppare le tante consonanze tra i due artisti a partire dal discorso sulle apparenze (Belpoliti), per aprire ulteriormente la riflessione sulla funzione generativa della fotografia nell'opera celatiana (Spunta) e sulla collaborazione con il pittore e fotografo Carlo Gajani (Gimmelli), letta in parallelo a quella con Ghirri (Spunta).

Il dialogo con Gajani precede quello con Ghirri ed è ancora per lo più inesplorato. Celati frequenta Gajani fin dagli anni Sessanta, collabora con lui a vari progetti negli anni Settanta, periodo in cui, come ci ricorda Fameli, «la sintonia tra i due autori emiliani [...] è fortissima» (Fameli 2017: 447), dati i comuni interessi sul corpo, sulla bagarre, i deliri psicotici, gli «oggetti soffici», la ritualità quotidiana. In «Il retrobottega dei mimi. Celati, Gajani e il racconto per immagini», Gabriele Gimmelli ripercorre gli scambi tra i due artisti sfruttando materiale dell'archivio Gajani e concentrandosi in particolare su due collaborazioni: Il chiodo in testa (1974) e La bottega dei mimi (1979). Basandosi sulla recente analisi dei due iconotesti proposta da Palmieri, Gimmelli sottolinea in questi testi una consonanza con le prime comiche celatiane, suggerendo come il «benjaminiano magazzino di scarti» in gioco nella collaborazione con Gajani e quella «necessità di "sfuggire" alla fissità della parola scritta» non tarderanno a tornare nella successiva vicenda artistica dello scrittore. "Gianni Celati e la fotografia come "pratico pensare per immagini" - in dialogo con Ghirri e Gajani» mette in dialogo il pensiero celatiano su Gajani e quello su Ghirri e getta luce su un tema ancora non trattato - l'incompiuto progetto sul paesaggio padano di Celati e Gajani. Nel suo saggio Marina Spunta discute la funzione generativa della fotografia nell'opera celatiana quale mezzo per aprirsi in modo nuovo alla visione del paesaggio negli anni Ottanta, per riflettere sulla (propria) scrittura e per rimodellare la propria immagine autoriale in quegli anni. Avvalendosi del materiale del Fondo Celati e del Fondo Gajani, e dell'analisi di testi editi e manoscritti di Verso la foce, Spunta dimostra come tali testi e riflessioni siano parte integrante del discorso celatiano sulle immagini e in linea con il pensiero ghirriano, quale tentativo di "pratico pensare per immagini». Nel saggio «Nella nebbia e nelle apparenze: Celati e Ghirri», Marco Belpoliti riprende il discorso sulle apparenze, introdotto nel volume da Bazzocchi, rileggendo due importanti scritti celatiani, «Finzioni a cui credere», del 1984, e «Commenti su un teatro naturale delle immagini», del 1989, testo quest'ultimo che fungerà da introduzione al fotolibro ghirriano Il profilo delle nuvole. Grazie a una lettura incrociata di questi testi e dell'opera ghirriana, la riflessione di Belpoliti ripercorre alcuni temi chiave celatiani, quali l'essere dispersi, la soglia d'intensità della visione, il «pensare-immaginare», l'esterno, e sviluppa il discorso sul vedere come «visione atmosferica» (cfr. Belpoliti 2016). L'autore 
rende evocativamente il senso dell'opera celatiana con queste parole: «Ci siamo persi nella nebbia, come i bambini pendolari, ci suggerisce Celati. Ma è solo perdendoci che potremo ritrovarci». Il saggio che conclude questo percorso sulla fotografia, apre al tempo stesso un'ulteriore sezione dei lavori. Cecilia Monina, in «Doppio framing, indugi e soglie narrative. Tre autori a confronto: Gianni Celati, Luigi Ghirri e Michelangelo Antonioni», sviluppa il tema del «doppio framing» tramite un confronto con la fotografia di Ghirri e il cinema di Antonioni. Monina ribadisce come la loro arte sia inscindibile da una riflessione dalla «visione con», del «vedere del vedere», quindi sulla cornice e su quello che resta fuori dal quadro. Ed è proprio la cornice, come spazio liminare, che invita lo spettatore a un dialogo con l'immagine. Monina rintraccia un procedimento simile nell'uso della voce narrante in Narratori delle pianure, testo imperniato sulla molteplicità delle voci e cornici narrative, piuttosto che sul narratore, come ci ricorda già Elena Porciani (2009).

La frequentazione celatiana con il cinema attraversa in forme diverse tutta la sua opera, in parallelo alla sua riflessione sulle arti visive e sulla fotografia: anima le prime narrazioni nella riscrittura dello slapstick dei fratelli Marx o Buster Keaton, nello «sguardo in macchina» dei personaggi, come ci ricorda Giulio Iacoli, è forza generatrice di molti racconti degli anni Ottanta e oltre, a partire dalla raccolta Cinema naturale, infine sfocia nei suoi documentari. Ed è nell'immagine di un "cinema naturale» della mente, come hanno sottolineato vari studiosi celatiani a partire da Rebecca West, che troviamo una chiave per il pensiero figurale di Celati e un nesso con una certa tradizione e riflessione sullo sguardo, la visione e la «fantasticazione» che va da Dante a Leopardi, da Calvino a Pasolini. In un'intervista con Sarah Patricia Hill, Celati sottolinea la novità di Rossellini nell'aver messo «in primo piano il problema percettivo, ossia d'un modo più naturale di guardare», ripensando «il cinema attraverso le risorse d'una visione documentaristica» - approccio che «dà via libera a un modo visionario di osservare tutto» (Celati in Hill 2003: 132-133). Come in Rossellini, così in Antonioni e in Ghirri la visione documentaria va di pari passo al lavoro dell'immaginazione, della fantasia, della creazione di «finzioni a cui credere» per darci sollievo. I cinque saggi che compongono la parte finale del volume affrontano questi temi da prospettive diverse e approcci disciplinari complementari - italianistica, comparatistica e studi di cinema - offrendo una panoramica a 360 gradi dell'opera celatiana.

Partendo dal discorso sul «cinema naturale» e dalla recensione a Narratori delle pianure di Guido Fink, collega di Celati a Bologna e specialista di letteratura e cinema angloamericani, che suggeriva uno sguardo agli antipodi del Palomar calviniano, in «Psichedelie visioni allucinazioni» Ugo Fracassa discute la «Sostenibilità/insostenibilità dello sguardo nell'opera di Celati». Basandosi su vari testi critici e narrativi dell'autore, dal saggio su Tozzi a Lunario del Paradiso a Fata Morgana, Fracassa giustappone il «vedere figurato della mente» che Celati teorizza nel saggio "Collezione di Spazi», al vedere retinico, superficiale, teso a delimitare contorni netti, che risponde alla posizione del realismo-naturalismo, 
sia in letteratura che nel cinema, spesso oggetto delle critiche celatiane. Il suo scopo è quello di rimettere assieme la bagarre del primo Celati e la distensione dello sguardo naturale sulle pianure, quindi «riconciliare motorio e visivo come i due poli di un unico universo poetico, del resto in gran parte scaturito dal camminar guardando». Giulio Iacoli in «Un palinsesto frastornante. Celati, la riscrittura dei fratelli Marx e dintorni» legge uno dei primi testi celatiani, $\mathrm{La}$ Farsa dei Tre Clandestini (1971-1972), tramite la lente della libera riscrittura delle slapstick comedies dei fratelli Marx, in particolare di Monkey Business, che Celati aveva tradotto. Integrando nel suo studio recenti teorie sulla riscrittura, l'adattamento e la trasposizione mediale, Iacoli legge il testo celatiano come traduzione-pastiche, dimostrando la felicità della riscrittura nonostante la difficoltà di rendere i giochi verbali. Michele Ronchi Stefanati riprende il tema dell'impegno politico nei documentari celatiani in «Cineamatori militanti. Ricadute politiche dei documentari di Gianni Celati». In linea con i saggi precedenti, l'autore vede una continuità nella scrittura celatiana ispirata al cinema, dai primi testi alle narrazioni naturali degli anni Ottanta, e sottolinea come nei documentari dell'autore ci si confronti tanto con un «metodo di osservazione del mondo esterno» quanto con una riflessione sul cinema come strumento politico che si contrappone ad alcuni fenomeni pervasivi della contemporaneità. Il saggio seguente, di Andrea Rondini, "Gianni Celati, il cinema di John Cassavetes e la distruzione dei dispositivi» legge l'opera di Celati in parallelo a quella del cineasta indipendente a lui coevo John Cassavetes, individuando un comune interesse per i marginali, gli outsiders, tra cui Celati pone anche l'autore, per le comiche, per l'improvvisazione come metodo dissipatorio. Rondini si sofferma in particolare su due film di Cassavetes citati da Celati in Conversazioni del vento volatore: Faces (1968), nel cui tessuto sonorofonico ibrido e destabilizzante intravede una consonanza con l'opera celatiana, e Husbands (1970), che celebra il personaggio errante nello spazio aperto, come in tanti scritti di Celati.

Il saggio conclusivo, di Denis Brotto, riporta infine l'attenzione su un elemento essenziale dei vagabondaggi artistici di Gianni Celati, quello dell'incontro, dello scambio, della relazione e dell'amicizia. Brotto accosta un'altra figura allo scrittore, in questo caso il poliedrico John Berger, amico di lunga data e collaboratore in vari progetti, tra cui il film Case sparse. Sfruttando lo scambio epistolare inedito conservato presso l'archivio Berger alla British Library di Londra, Brotto mette in luce una profonda consonanza di vedute, in particolare sulla scrittura come forma terapica, sulla narrazione come «inner voice», sulla lingua come forma di accoglienza, ospitalità, e antidoto agli stati depressivi. La similarità della loro grafia a cui allude il titolo del saggio, «Di una medesima grafia. Lo scambio epistolare tra Gianni Celati e John Berger (19921997)», rafforza quest'idea di consonanza di vedute che spazia dall'influenza benjaminiana, al pensiero archeologico, al ruolo dell'arte nel contemporaneo.

In un breve saggio su Ghirri, dal titolo, «Per un piccolo catalogo di immagini», Giorgio Messori ci ricorda che: 
Per guardare le foto di Ghirri bisogna invece predisporsi in modo diverso, perché non dobbiamo cercare solo degli effetti immediati. Bisognerebbe trovare la stessa calma che ci è indispensabile, ad esempio, quando ci accingiamo a leggere un libro. Allora potremmo seguire le sue immagini e scoprire con lui, nel libero gioco delle analogie, quel gioco infinito del mondo che può sempre rivelare somiglianze inattese, somiglianze che vanno oltre le categorie di giudizio e di grandezza, di ciò che è vicino o lontano, remoto. E questo è anche il modo per ritrovare un senso di appartenenza altrimenti perduto. Un po' come nelle palme in riva al mare, che poi ritornano stampate, in unaltra immagine, su una camicia stile hawayano. (Messori 1995: s.p.)

In quest'ottica vorremmo affidarci alla riflessione celatiana sullo sguardo e sul «vedere del vedere» come un'apertura al «libero gioco delle analogie» di cui parla Messori, che può far nascere inattese somiglianze tra arti diverse, e dar vita a un qualcosa che sfugge e va oltre l'evidenza della superficie, e che apra l'opera di Celati a nuove letture e nuovi sensi.

\section{Bibliografia}

Belpoliti M., 2016, "Gianni Celati, la letteratura in bilico sull'abisso», in Celati G., Romanzi, cronache e racconti, a cura di M. Belpoliti e N. Palmieri, Milano, Mondadori, p. XI-LXXII.

Celati G., 2008, «Viaggio in Italia con 20 fotografi, 20 anni dopo», in Gianni Celati, Riga, 28, a cura di M. Belpoliti e M. Sironi, p. 126-135.

Celati G., 2011, Conversazioni del vento volatore, Macerata, Quodlibet.

Celati G., 2017, Animazioni e incantamenti, con C. Gajani, a cura di N. Palmieri, Roma, L'Orma.

Celati G., s.d., «La media oscurità dell'esperienza», busta 1/2, Fondo Celati, Reggio Emilia, Biblioteca Panizzi, 20 carte sciolte.

Deleuze G., 1968, Différence et répétition, Paris, Presses universitaires de France.

Didi-Huberman G., 1992, Ce que nous voyons, ce qui nous regarde, Paris, Les Éditions de Minuit.

Fameli P., 2017, "Celati e Gajani in dialogo», in Celati 2017, p. 441-451.

Ghirri L., Leone G. e Velati E. (a cura di), 1984, Viaggio in Italia, Alessandria, Il Quadrante.

Hill S., 2003, «Documentari imprevedibili come i sogni. Conversazione tra Gianni Celati e Sarah Hill», in L'idea documentaria, a cura di M. Bertozzi, Torino, Lindau, p. 129-139.

Martelli M., 2019, L'impensé du regard. Trois études sur Gianni Celati et les arts visuels, Macerata, Quodlibet.

Messori G., 1995, «Per un piccolo catalogo di immagini», in Luigi Ghirri. Per un piccolo catalogo di immagini, Castelvetro Piacentino, Nuova Lito Effe.

Nancy J.-L., 2002, À l'écoute, Paris, Galilée. 
Palmieri N., 2017, «Il gesto, gli oggetti, le immagini. Gianni Celati nell’animazione del mondo», in Celati 2017, p. 421-438.

Porciani E., 2009, «Le cornici invisibili di Narratori delle pianure», in Letteratura come fantasticazione. In conversazione con Gianni Celati, a cura di L. Rorato e M. Spunta, Lampeter, Edwin Mellen Press, p. 279-290.

Spunta M. e Benci J. (a cura di), 2017, Luigi Ghirri and the Photography of Place. Interdisciplinary Perspectives, Oxford, Peter Lang.

Valtorta R., 2013, «In cerca di luoghi (non si trattava solo di paesaggio)», in Luogo e identità nella fotografia italiana contemporanea, a cura di R. Valtorta, Torino, Einaudi, p. 3-108.

West R., 2000, Gianni Celati. The craft of everyday storytelling, Toronto, University of Toronto Press. 